\title{
Tree-Based Classification Algorithm for Heterogeneous Unique Item ID Schemes
}

\author{
Yong Hwan Lee ${ }^{1}$, Hee Jung Kim ${ }^{1}$, Byeong-hee Roh ${ }^{1}$, S.W. Yoo ${ }^{1}$, and Y.C. Oh ${ }^{2}$ \\ 1 Graduate School of Information and Communication, Ajou University, \\ San 5 Wonchon-dong, Youngtong-Gu, Suwon, 443-749, Korea \\ \{lnj486, rla81, bhroh, swyoo\}@ajou.ac.kr \\ 2 Samsung Electronics Cooperation, Suwon, Korea \\ ycoh@samsung.com
}

\begin{abstract}
For RFID-based applications, the uniqueness of ID assigned to each RFID tag should be guaranteed. Several research/standard organizations such as EPCglobal, ISO/IEC, Ubiquitous ID Center, and so on, have developed their own Unique Item ID (UII) specifications. The existence of various UII schemes may cause interoperability problems between applications using different UII schemes when those applications are operated on future global Internet network environment. In addition, it is expected that the traffic for UII query will be increased ten-times higher than that for DNS query in the current Internet. In order to overcome these problems, this paper proposes a fast tree-based classification algorithm applicable for various UII schemes, which can make it efficient to construct global directory lookup services for RFID applications with various UII schemes. Since the proposed scheme can be operated on readers, it can not only distribute traffic loads for UII queries, but also global RFID networks.
\end{abstract}

\section{Introduction}

Radio Frequency Identification (RFID) is a technology for the applications based on identification of the physical objects used for tracking and finding locations of objects. For RFID-related applications and services, the uniqueness of the identification assigned to each object should be guaranteed. Unique Item ID (UII) is an identification that uniquely identifies a specific object during its life.

There have been several standardization works for RFID UII by large research organizations. MIT Auto-ID Center developed a UII scheme called Electronic Product Code (EPC) [1, which is evolved from barcode systems widely used in supply-chains and management of manufactures. There are two types of UII schemes with ID lengths of 64-bit and 96-bit, respectively. Ubiquitous ID Center in Japan also developed its own UII scheme, called Ubiquitous Code (ucode) 2]. The ucode is the 128-bit long and can be extended as needed in 128-bit units such as 256, 384, or 512-bit. The ucode is a meta-code system so that it can be used to various kind of identification-based systems and services applied to intangible things such as services, softwares and so on, as well as materials made 
by manufactures. ISO/IEC also developed ISO/IEC15459 series as UII schemes for RFID 3] 4] 5]. In ISO/IEC's UII scheme, each ID is represented by one to four numbers or characters using ASCII values 6 . Under the existence of various UII schemes currently as above, another works for developing new UII schemes such as [7] are being carried out. Likewise, it is expected that there will be various and complicated UII schemes.

The existence of various UII schemes may cause interoperability problems between applications using different UII schemes when those applications are operated on future global Internet network environment. Under global network environments, all UII query operations are done in public network infrastructures such as the current or future Internet, not in intra or closed network domains. The procedure for UII query is as similar as DNS query in the Internet. However, it is expected that the traffic for UII query will be increased ten-times higher than that for DNS. Under the circumstances, lookup speed for UII query can be a primary factor for the success of RFID-related services. EPCgloagl developed Object Name Service (ONS) for providing a global lookup service to translate an EPC UII into one or more Internet URLs which often identify an EPC information service [8]. However, the lookup service of ONS can support EPC's UII scheme only. In order to support global UII lookup services independent of any specific UII schemes, Multi-code Directory Service(MDS) has been developed by National Internet Development Agency(NIDA) of Korea 9. MDS has been being operated as a trial system for RFID-based applications and services under public Internet environment in Korea since January 2005. To support global directory service for various UII schemes, MDS uses its own classification algorithm. The algorithm, for each UII query request, classifies what kind of UII scheme is necessary for the resolution of the request. Then, MDS sends the request to appropriate resolving server. However, since the classification algorithm used in MDS is done according to a given priority to a specific UII scheme such as EPC first, its performance decreases as the occupancy ratio specific UII scheme decreases. In addition, all classification processes for UII queries are done in MDS, the burden for operating the classification algorithm in MDS increases as those query requests increase.

This paper proposes a fast and efficient tree-based classification algorithm of UII schemes for global UII directory lookup services. As in MDS, the proposed algorithm is used to determine the appropriate UII scheme necessary for resolving each UII query request. It is noted that unlike DNS query request, RFID tags can provide only ID information consisting of binary bits. Since RFID applications need to know some additional information related to each tag ID, they have to ask it to UII resolving server such as EPCIS (EPC Information Service). However, with tag ID itself, it can not be known what UII resolving server it can ask to under the environments where various UII schemes are working together. The proposed algorithm can solve the problem with the following features. First, it guarantees a constant performance regardless of the types of UII schemes and their distributions, unlike the algorithm used in MDS whose performance depends on the penetration ratio of specific UII scheme. Second, it 
can be operated at each reader, it can not only decentralize traffic loads for UII queries, but also make it easy to manage global RFID networks.

The paper is organized as follows. In Section 2, we briefly explain some related works such as existing UII schemes and the classification algorithm used in MDS. The proposed algorithm and its performance results are presented in Section 3 and Section 4, respectively. Finally, we conclude the paper in Section 5.

\section{Related Works}

\subsection{Unique Item ID (UII)}

EPCglobal. The general structure of the Electronic Product Code (EPC) consists of a fixed length header and a series of numbers, as illustrated in Fig [1(a) 1]. The Numbers field comprises three entities such as Domain Manager, Object Class, and Serial Number. The substructure of the entities of the Numbers field and its own pertinent role are determined by the header value. The Domain Manager identifies the EPC manager of the UII. This field is assigned to institutions or companies responsible for maintaining the Object Class and Serial Number. The Object Class indicates the group or class of the object, and the Domain Manager must guarantee unique assignment of Object Class numbers within its own managing domain. The Serial Number is a specific number for every products and should be uniquely assigned within the range of the Object Class.

ISO/IEC. In Fig 1(b), the general UII structure defined by ISO/IEC 15459 is shown 3] 4] 5]. In SI (Symbology Identifier), the type of data format defined by AIM (association for Automatic Identification and Mobility) is written. SI can be used for decoding the data expression of UII. It is noted that SI is not included in RFID tag ID. After reader reads the tag ID, SI is optionally added into the UII by the reader. The Class Identification field indicates the class of the UID (Unique IDentifier). The class information is used to identify products according to the specific rule defined for each class. The UID is an identifier uniquely assigned to every individual product by the issuing agency.

Ubiquitous ID Center. The ucode (ubiquitous Code) is an UII scheme developed by the ubiquitous ID center in Japan. The length of the ucode is basically 128-bit long, and can be extended as needed in 128-bit units such as 256-bit, 384 or 512 -bit 9. Since the ucode adopts the meta-code system, it can be used to various kind of identification-based systems and services applied to intangible things such as services, softwares and so on, as well as materials made by manufactures. The 128-bit length of ucode can accommodate ISBN (International Standard Bibliographic Number), ISSN (International Standard Serial Number) publication ID, and existing barcode scheme. And, it can accommodate IPv6 addresses. An example of ucode structure is shown in Fig 1(c), in which it can accommodate all barcodes used in Japan by encoding the JAN (Japan Article Number) code. Code Identifier indicates the type of code used in the ucode. In 


\begin{tabular}{|l|l|l|}
\hline Header & \multicolumn{2}{|c|}{ Numbers } \\
\hline
\end{tabular}

(a)

\begin{tabular}{|l|c|c|}
\hline Symbology & Class Identification & Unique Identifier (UID) \\
\hline \multicolumn{4}{|c|}{ (b) } \\
\begin{tabular}{|l|l|c|}
\hline \multicolumn{4}{|c|}{63} & 64 & Unique ID \\
\hline Code Identifier & JAN code segment & Uniq \\
\hline
\end{tabular}
\end{tabular}

(c)

Fig. 1. Various UII schemes (a) EPC UII format example (b)general UII structure of ISO/IEC (c) ucode example

the example, Code Identifier indicates that the following code is used for the JAN code. As in the example shown in Fig[1(c), where JAN code is used, Unique ID with 64-bit long identifies individual items uniquely distinguished from others.

\subsection{Multi-code Directory Service(MDS)}

As mentioned above, the existence of various UII schemes and the expected rapid growth of UII query traffic in future global RFID network environments may cause severe problems in managing and deploying RFID services and networks. Under such circumstances with heterogeneous UII schemes, fast global lookup for UII query can be a primary factor for the success of RFID-related services.

MDS. MDS 9 has been developed for the global RFID directory services to provide interoperability between applications and services with different UII schemes under the public Internet environment, especially, EPC, ISO/IEC and ucode. MDS has been being operated as a trial system in Korea since January 2005. The purpose of MDS is to implement global RFID network environment independent of UII schemes.

Classification Algorithm Used in MDS. RFID tags can provide only ID information consisting of binary bits. Since RFID applications need to know some additional information related to each tag ID, they have to ask it to UII resolving server such as EPC's ONS. However, with tag ID itself, it is impossible to know what UII resolving server they can ask to under the environments where various UII schemes are using together. MDS solves the problem as follows. All readers send UII query messages to MDS. Then, the classification module used in MDS figures out what UII scheme is used in the tag ID. According to the result from the classification module, MDS send the query message to corresponding UII resolving servers in behalf of readers. Then, the resolving message can be sent back to readers through MDS.

Fig 2 shows the procedure of the classification algorithm used in MDS. In MDS, it is assumed that the standardization processes in EPCglobal and ISO/ 


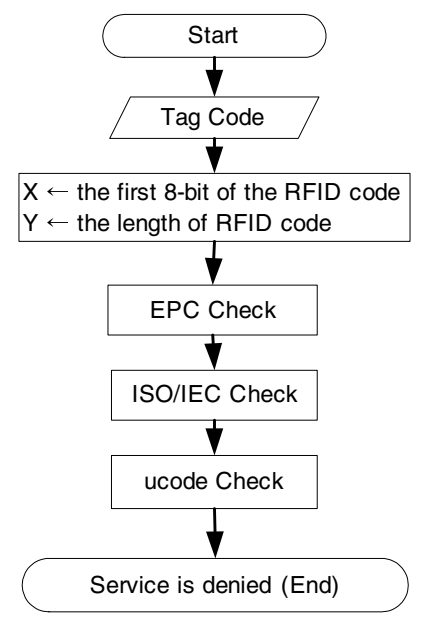

Fig. 2. Basic process of classification algorithm used in MDS

IEC are faster than others, and these UII schemes of two groups will be used on commercial business earlier. From the expectation, as shown in Fig, the classification algorithm used in MDS is done according to a given priority to a specific UII scheme such as EPC first, ISO/IEC next, and finally ucode. Accordingly, the algorithm has some weakness that its performance decreases as the occupancy ratio of specific UII scheme decreases. In addition, since all classification processes for UII queries are done in MDS, the burden for operating the classification algorithm in MDS increases as those query requests increase.

\section{Tree-Based Classification Algorithm}

In this Section, we explain our proposed tree-based UII classification algorithm. As shown in Fig 3, we can make a binary tree for classifying between EPC and ISO/IEC's UII schemes according to the specifications of them. When it is necessary for a new UII scheme to be added, we can easily add the new scheme into the tree as in normal binary tree manipulation process. In the tree shown in Fig 3. except for the only case when initial two bits of header are '10', there is no confusion to classify between UII schemes. That is, following to the binary tree, these UII schemes can be classified simply when initial two initial bits are not '10'. In case when two initial bits are '10', it needs additional process to classify between the two schemes, SGTIN-64 of EPC and Alphabet UII of ISO/IEC. In this case, by comparing the length and the consistency in the fields of the UII as illustrated in Section 2, the two schemes can be easily classified.

To speed up the classification process, instead of comparing all bits as in MDS, only necessary minimum bits are used for the classification. Except for ISO/IEC's UII and EPC's SGTIN-64 UII scheme, other EPC's UII schemes are 


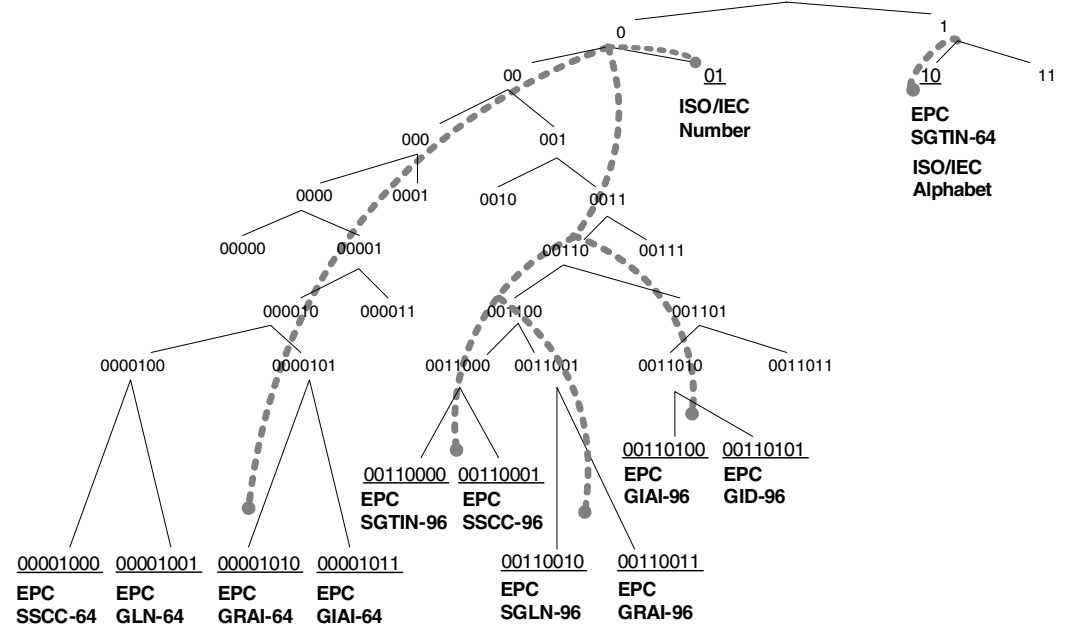

Fig. 3. Tree architecture to classify UIIs

used '00' as their initial two bits. With the UII codes using '00' as their initial two bits, we can classify those UII schemes by comparing only four bits as shown in Fig 4. That is, by windowing the four bits and comparing the bit values in the window, we can easily classify those UII schemes.

In Fig [5, the overall classification process of the proposed algorithm is shown as a pseudo-code style. In the pseudo-code, it is assumed that in all UII codes, there are only valid EPC or ISO/IEC UIIs. When the first bit is '1', next second bit should be '0' according to our assumption. In this case, the UII is one of ISO/IEC's alphabet or EPC's SGTIN-64 UII schemes. The two schemes are easily classified by comparing the UII code length. That is, if the UII code length is equal to 64, the UII is EPC's SGTIN-64, otherwise ISO/IEC's alphabet. When two initial bits are '01', it is classified UII scheme of ISO/IEC corresponding to number. The other UII bits corresponds to EPC-related UII schemes, and to classify these UII schemes, four bits as shown in Fig 4 are compared.

Fig [ 6 illustrates the outline of the proposed method and the method used in MDS. In MDS shown in Fig[6(a), all UII queries are sent to MDS and their corresponding URI are translated by using its classification method in MDS. Then, MDS sends the URI query to corresponding directory servers such as National ODS (Object Directory System) operated by NIDA, ONS by EPC, and so on, for getting the additional information. Likewise, since all query processes are done in MDS in a centralized way, it can be applied only when there are very low query traffic loads. That is, in case when query traffic increases exponentially in future RFID global networks, the MDS-based scheme may have limitations. On the contrary, as shown in Fig 6(b), the URI translation as a result of the classification of UII scheme can be done at readers. This feature can be obtained because the proposed algorithm can be done with very low computational com- 


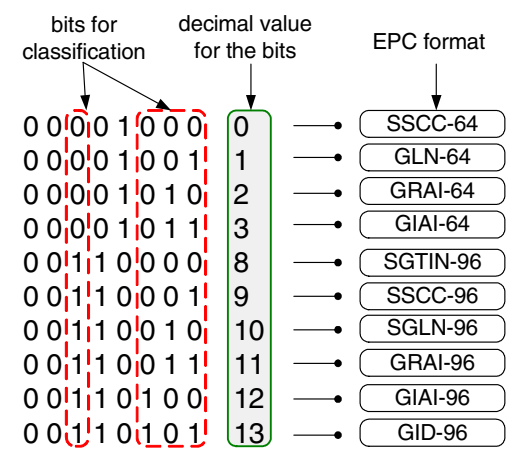

Fig. 4. Bits for classifying EPC's UII schemes

Table 1. Comparisons of algorithm used for the experiment

\begin{tabular}{|c|c|c|c|}
\hline Algorithm & MDS-A & MDS-C & PROPOSED \\
\hline Process & Priority-based & Priority-based & Bit information \\
\hline Initial assumption & 8-bit header/length & 8-bit header/length & nothing \\
\hline Procedure(EPC) & $\begin{array}{c}\text { 1.header(EPC-64) } \\
\text { 2.length }\end{array}$ & $\begin{array}{c}\text { 1.length(EPC-96) } \\
\text { Bit sequence }\end{array}$ & 2.header \\
\hline Procedure(ISO) & $\begin{array}{c}\text { Check if the header includes numbers } \\
\text { or characters in leading 8-bit }\end{array}$ & Bit sequence \\
\hline Error handling & \multicolumn{3}{|c|}{ Out of consideration } \\
\hline
\end{tabular}

plexity. Accordingly, it can not only decentralize traffic loads for UII queries, but also make it easy to manage global RFID networks.

\section{Experimental Results}

For the experiments, UIIs are generated randomly according to EPC and ISO/ IEC 15459 specifications. To show the efficiency of the proposed algorithm, we compared its performances with those of algorithms used in MDS. The features of those comparable schemes are shown in Table 1. In Table 1, whereas PROPOSED denotes our proposed algorithm, MDS-A and MDS-C are the classification algorithms used in MDS[10]. MDS-A and MDS-C have different priority strategies to classify UII schemes as shown in Table1. Especially, MDS-C is working in the MDS trial service operated by NIDA. In MDS-A and MDS-C, it is assumed that the two algorithms already know the 8-bit header information and the length of the UII before the classification[10. However, the PROPOSED performs without the knowledge of them, and its classification process is done based on the bit-by-bit operation on tag IDs.

Accuracy of Classification. In order to check the accuracy of the algorithms, we made artificial UIIs according to corresponding schemes, and mixed them randomly. It is noted that there are only valid UIIs in the mixed sequences. 


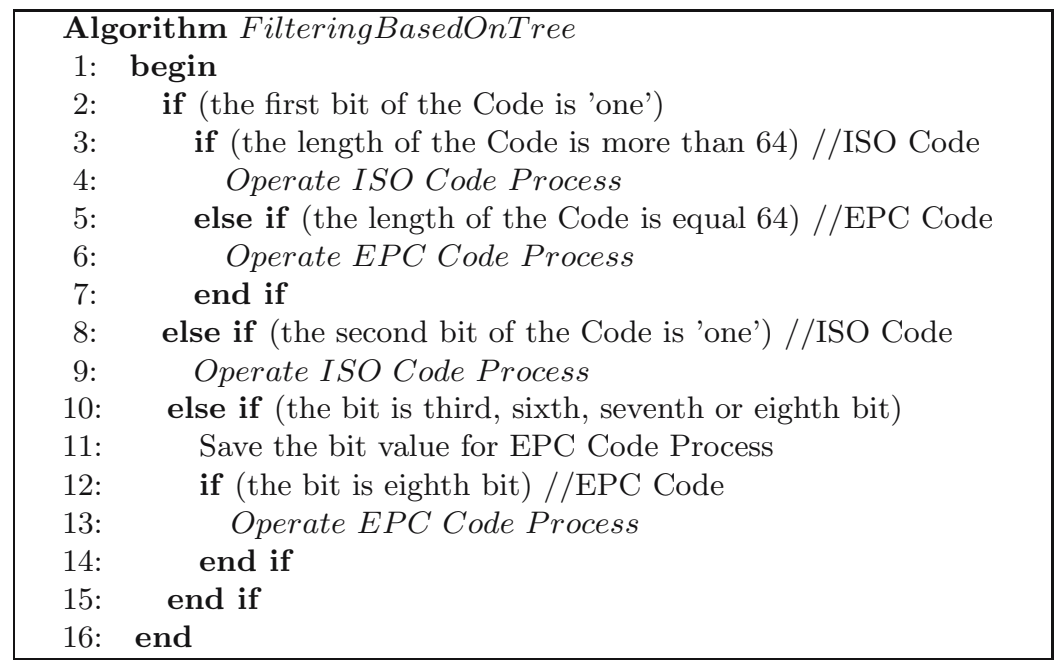

Fig. 5. Pseudo code of the proposed algorithm

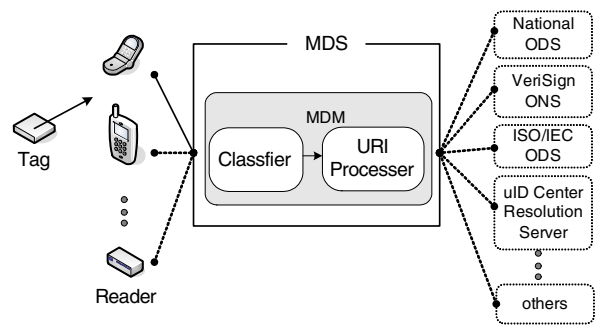

(a)

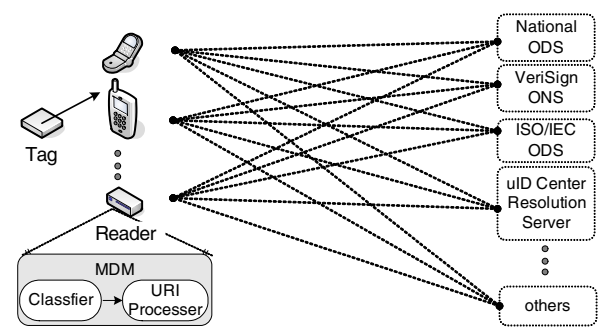

(b)

Fig. 6. Application architectures of (a) MDS and (b) proposed scheme

Then, we tested the classification algorithms using the mixed UII sequences, and found that there was no failure in classification by all the algorithms.

Effect on Length of EPC UII. As mentioned before, different UII schemes may have different code lengths. For example, the length of each EPC UII is either 64-bit (EPC-64) or 96-bit (EPC-96). In order to find out how the algorithms are affected by the various lengths of UII schemes, we carried out the following experiment. First, we randomly generated 10000 ISO/IEC UIIs and 10000 EPC UIIs varying the ratio between EPC-64 and EPC-96. Then, we mixed the UIIs randomly. Fig 7 shows the processing time for classifying all UIIs using each algorithm varying the occupancy ratio of the EPC-96 to EPC-64. Each algorithm was implemented by using $\mathrm{C}$ language, and we executed those algorithms on the computer of a Pentium-4 2.80GHz with Windows XP OS. We carried out 100 times of experiments, and its average values are shown in Fig:7(a). In the horizontal axis of Fig 7 (a), the value $20 \%$ means there are $20 \%$ EPC-96 


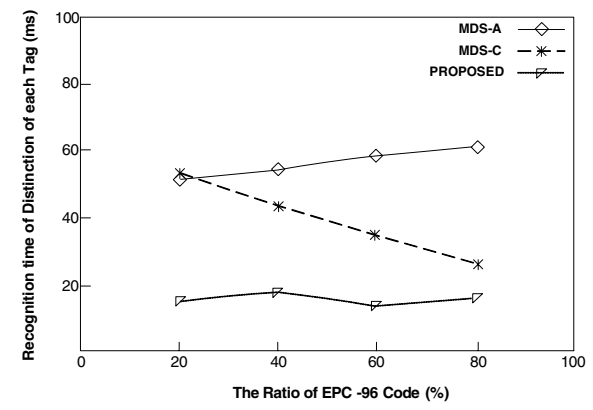

(a)

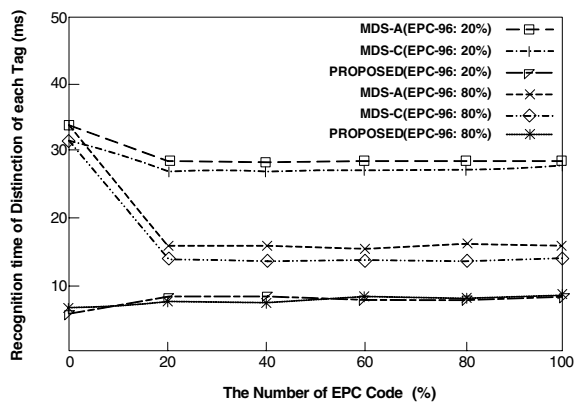

(b)

Fig. 7. Processing time varying (a) the ratio of EPC-96 (b)the occupancy ratio between UII schemes

UIIs out of total 10000 EPC UIIs while ISO/IEC's UIIs are constant at 10000 . As mentioned before, it is noted that MDS algorithms perform the classification with higher priority to EPC UIIs, but MDS-A handles the EPC-64 scheme first while MDS-C does the EPC-96 first. As the ratio of EPC-96 increases, the processing time of MDS-A increases also, while the processing time of MDS-C decreases. However, the proposed algorithm provides constant performances independent of the variance of the ratio of EPC-96. And, the proposed algorithm shows much lower processing time performances than MDS-A and MDS-C. It is expected that in future RFID network environments EPC-96 will be used much more than EPC-64. This experiment show that the proposed algorithm can be applied to both current and future global RFID network environments more efficiently than MDS algorithms.

Effect on Occupancy Ratio of UII Schemes. Under the existence of various UII schemes, the performance varying the occupancy ratio of UII schemes is one of the most important factors to compare the classification algorithms. In terms of performance, especially, if the performances of algorithms depend on the occupancy ratio of certain UII schemes, it may cause severe problems in managing RFID services and networks. In Fig:7(b), we show the performances of the comparable algorithms by varying the occupancy ratio of UII schemes. For obtaining Fig[7(b), we generated total 10000 UIIs. Among the 10000 UIIs, we varied the ratio of EPC UIIs from $0 \%$ where there are only ISO/IEC UIIs to $100 \%$ where no ISO/IEC UIIs exist. Among EPC UIIs, there are two cases when the ratios of EPC-96 are fixed at 20\% and 80\%. These two cases are reflecting the environments of RFID applications in the current and future RFID networks. In future RFID networks, it is expected that more EPC-96 UIIs are used than EPC-64. From Fig:7(b), the occupancy ratio between UII schemes can affect the performances of algorithms MDS-A and MDS-C. This phenomenon can be explained as same as in the case of Fig. 71(a). However, the proposed algorithm shows much lower processing time than MDS algorithms independent of the occupancy ratios of UII schemes. 


\section{Conclusion}

In this paper, we proposed a fast and efficient tree-based algorithm for classifying various UII schemes in global UII directory lookup services. We show that the proposed algorithm can provide constant performances independent of UII schemes and lower processing time than the current MDS algorithms. And, the proposed algorithm can be operated at each reader, it can not only decentralize traffic loads for UII queries, but also make it easy to manage global RFID networks. Though we explained our proposed scheme in the case for two UII schemes such as EPC and ISO/IEC, it can be easily extended to all kind of UII schemes by adding new required UII schemes into the tree.

It is expected that the traffic for query will be increased ten-times higher than that for DNS. Under the circumstances, lookup speed for UII query can be a primary factor for the success of RFID-related services. The main features of the proposed algorithm such as faster speed, the independence of UII schemes and the operation at readers can be applied in global RFID network environments.

\section{Acknowledgement}

This work was supported by grant (No. 05A3-I3-10) from Ubiquitous Autonomic Computing and Network Project sponsored by the Ministry of Information and Communication, Korea.

\section{References}

1. EPCglobal, "EPC Tag Data Standard Version 1.1 Rev.1.26," Nov. 2004

2. Ken Sakamura, "Ubiquitous ID Center has authorized 2 types of RFID chips made by Fujitsu as the Standard ucode tag," uID Center, Dec. 2004

3. ISO/IEC 15459-1, "Information technology-Unique identifiers for item management Part 1: Unique identification of transport unit," Sep. 2004

4. ISO/IEC 15459-3, "Information technology-Unique identifiers for item management Part 3: Common rules for unique identification," Sep. 2004

5. ISO/IEC 15459-4," Information technology-Unique identifiers for item management Part 4: Unique item identification for supply chain management," Sep. 2004

6. Material Handling Industry, "Data Identifier and Application Identifier Standard, Standard Under Continuous Maintenance," Jun. 2004

7. KISTI:, "China, Global RFID standard development propulsion," Overseas scientific technical trend TSTA200510135367, Mar. 2005

8. Auto-ID Center, "Auto-ID Object Name Service (ONS) 1.0," Aug. 2003

9. NIDA, "MDS Guide Line V1.0," Guide Manual, Dec. 2004

10. S.W. Yoo, et al., "Registration and Management in RFID ONS and Policy Research," Final Report, NIDA, Nov. 2004 